\title{
Transmission Kikuchi Diffraction (TKD)via a horizontally positioned detector.
}

\author{
Fundenberger J.-J. ${ }^{1,2}$, Bouzy E. ${ }^{1,2}$, Goran D. ${ }^{3}$, Guyon J. ${ }^{1,2}$, Morawiec A. ${ }^{4}$, Yuan H. ${ }^{1,2}$ \\ ${ }^{1}$ Laboratoire d'Etude des Microstructures et de Mécanique des Matériaux (LEM3), UMR CNRS \\ 7239,Université de Lorraine, 57045 Metz, France \\ 2.Laboratory of Excellence on Design of Alloy Metals for low-MAss Structures (DAMAS), Université \\ de Lorraine,57045 Metz, France \\ ${ }^{3}$ Bruker Nano GmbH, Am Studio 2D,12489 Berlin, Germany \\ 4.Institute of Metallurgy and Materials Science, Polish Academy of Sciences, Reymonta 25, 30059 \\ Krakow, Poland
}

Orientation mapping techniques are become indispensable tools for materials characterization. Indeed, they allow for quantification of phase amounts, local crystallographic textures, grain boundary characters, grain sizes, densities of geometrical necessary dislocations et caetera. In particular, the EBSD mapping technique has been a tremendous success because it helps in quick characterization of bulk crystalline materials. However, its lateral spatial resolution is limited. Moreover, as the specimen is highly tilted during the EBSD measurement, the resolution is 'anisotropic': it is three times worse in the direction perpendicular to the tilt axis than in the direction of the axis. Consequently, this method is not suitable for application to ultrafine or nano-grained materials. One alternative is to use transmission electron microscopy (TEM) to get orientation maps from Kikuchi patterns using large angle convergent nano-beam [1] or from spot patterns using low angle nano-beam with precession [2]; the lateral resolution of these techniques is in the range of 5-10nm. Recent developments in orientation mapping based on SEM in transmission mode (labeled TKD for Transmission Kikuchi Diffraction) pushed the lateral resolution to about $10 \mathrm{~nm}$, that is nearly to the level of TEM-based techniques [3]. With TKD, high resolution maps are obtained using the same hardware and software as in standard EBSD systems [4]. In this presentation we will describe a new configuration for the TKD technique, and we will show that it allows us to achieve a lateral resolution even better than that of the conventional configuration.

The novel feature of our configuration is the position of phosphor scintillator. In the conventional TKD, it is positioned nearly vertically, whereas we positioned it horizontally. The scintillator was removed from the nose of the EBSD camera housing, and a mirror was placed to transfer the light emitted by the scintillator to the camera (see figure 1).The mirror is positioned so the optics of the camera is focussed on the scintillator. A thin foil is mounted on a $\mathrm{C}$-shaped transmission sample holder which is on a threeaxis mechanical stage. With this device, both the working distance and the sample-scintillator are independently adjustable. The Kikuchi patterns are free of any geometrical distortion. They are processed either on-line using commercial software or off-line using an in-house software originally developed for TEM-based orientation mapping [1].

Various high resolution orientation maps with a step size of $5 \mathrm{~nm}$ will be shown as they were obtained, i.e., without any clean-up or noise reduction. For example, an ultrafine grained silicon specimen with 10nm-wide twins inside the grains. The twins are clearly distinguishable on the recorded orientation map.

Lateral resolution tests have been carried out on a titanium aluminide with a lamellar microstructure. This specimen has been prepared using EBSD and FIB so the interfaces are edge-on. One-nanometer 
step size scans have been made through the interfaces using an electron beam current of $300 \mathrm{pA}$. The tests show that the physical lateral resolution, i.e. the minimum distance for which only one Kikuchi pattern is discernable, is about $5 \mathrm{~nm}$. The effective lateral resolution, which takes into account the ability of the indexing software to disentangle superimposed Kikuchi patterns, is even better than that value.

The reason for which our system with on-axis detection leads to an improved lateral resolution compared to the conventional system is that the intensity of inelastic electron scattering decreases with increasing scattering angle. Hence, more intense Kikuchi patterns are expected at low scattering angles. Consequently, smaller electron probe size can be used, and solvable patterns are obtained from smaller electron-material interaction volumes.

Summarizing, a conceptually simple and flexible configuration with on-axis detector of transmission Kikuchi patterns is presented. It allows us to achieve lateral resolution better than five nanometers. Due to this feature, the new TKD configuration seems ideal for investigating nano-grained materials.

References:

[1] Fundenberger J.J., Morawiec A., Bouzy E., Lecomte J.S. Ultramicroscopy 96(2003),p. 127.

[2] E.F. Rauch, M. Véron, J. Portillo, D. Bultreys, Y. Maniette, S. Nicolopoulos, Microscopy and Analysis 22 (2008) S5-S8.

[3] Keller R.R., Geiss R.H., Journal of Microscopy 245 (2012), p.245.

[4] Trimby P.W. Ultramicroscopy 120 (2012), p.16.

[5]Funderberger J.J., Bouzy E., Goran D., Guyon J., Yuan H., Morawiec A. submitted to Ultramicroscopy.

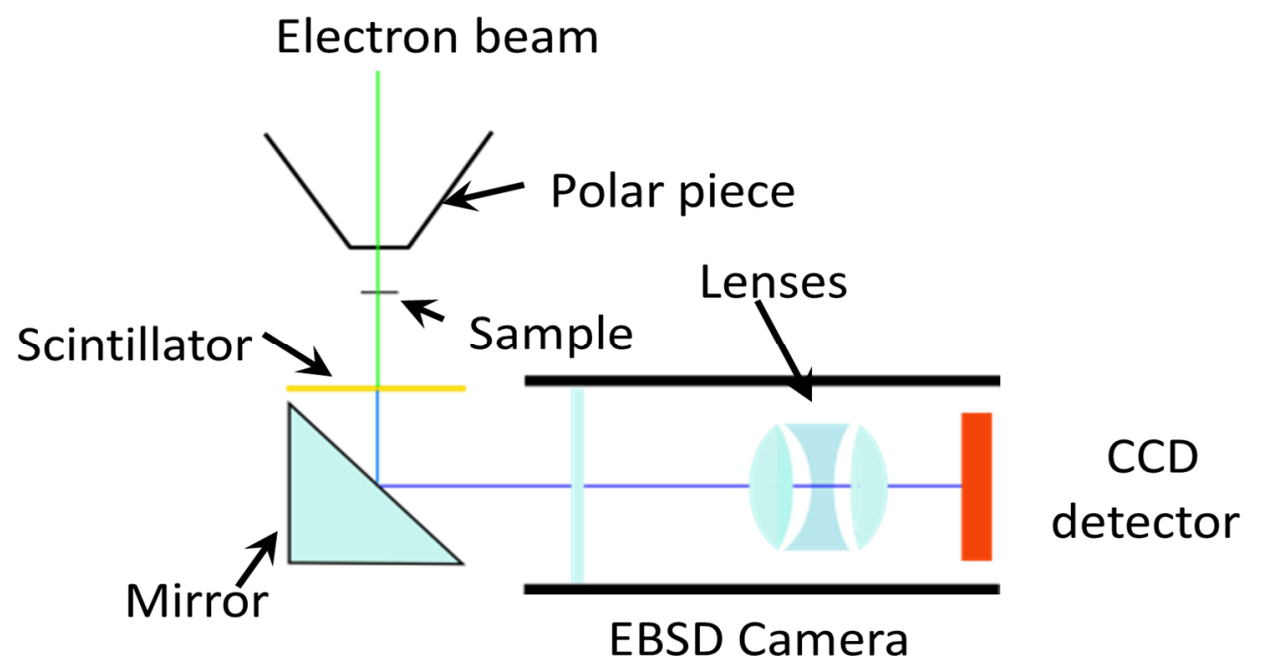

Figure 1. Schematic of the new TKD configuration with horizontally positioned detector. 